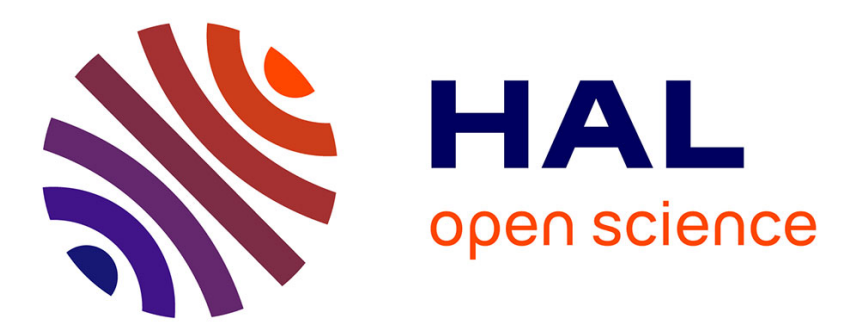

\title{
Analysis of Defect in Extreme UV Lithography Mask Using a Modal Method Based on Nodal B-Spline Expansion
}

Kofi Edee, Patrick Schiavone, Gérard Granet

\section{- To cite this version:}

Kofi Edee, Patrick Schiavone, Gérard Granet. Analysis of Defect in Extreme UV Lithography Mask Using a Modal Method Based on Nodal B-Spline Expansion. Japanese Journal of Applied Physics, part 1: Regular papers, Short Notes, 2005, Vol. 44, No. 9A, pp.6458-6462. 10.1143/JJAP.44.6458 . hal-00020645

\section{HAL Id: hal-00020645 https://hal.science/hal-00020645}

Submitted on 13 Mar 2006

HAL is a multi-disciplinary open access archive for the deposit and dissemination of scientific research documents, whether they are published or not. The documents may come from teaching and research institutions in France or abroad, or from public or private research centers.
L'archive ouverte pluridisciplinaire HAL, est destinée au dépôt et à la diffusion de documents scientifiques de niveau recherche, publiés ou non, émanant des établissements d'enseignement et de recherche français ou étrangers, des laboratoires publics ou privés. 
Analysis of defect in E.U.V. lithography mask using a Modal Method by Nodal B-Spline Expansion (MMSNE).

K. Edee*, P. Schiavone*, G. Granet**.

*Laboratoire des Technologies de la Microélectronique, CNRS, c/o CEA Grenoble, 17 rue des Martyrs 38054 Grenoble Cedex 09, France.

**Laboratoire des Sciences et Matériaux pour 1' Electronique et d' Automatisme, CNRS/ UMR, 6602, Université Blaise Pascal, Les Cézeaux 63177 Aubière Cedex. 


\section{Abstrat.}

This paper is devoted to an electromagnetic modeling of a lithography mask in E.U.V. For that purpose, a modal method based on a spline nodal expansion is presented. The results are successfully compared with those obtained from the F.D.T.D. (Finite Difference method in Time Domain) and from other modal method such as M.M.F.E. (Modal Method by Fourier Expansion) The spline nodal basis function implemented in this paper is the first step toward a multiresolution scheme that is expected to perform much more efficiently. 


\section{Introduction}

The aim of the electromagnetic modeling of the Extreme UV lithography mask is the study of the interaction between the electromagnetic field and the mask or a possible defect within or on top of the multilayer mirror. The EUV mask is a Bragg mirror which is made of fourty sequence of $\mathrm{Mo} / \mathrm{Si}$ (with refractive index $v=0.9227-0.0062 \mathrm{i} / v=0.9999-0.0018 \mathrm{i}$ ) bilayer, called the blank, under an $\mathrm{Cr}$ absorber. The structure is supposed to be illuminated by a plane wave. We denote by $k=2 \pi / \lambda$ the wave number ( $\lambda$ is the wavelength) and $\theta_{0}$ the incident angle. Time dependence is expressed by the factor $\exp (i \omega t)$ ( $\omega$ is the angular frequency). The canonical problem is the so-called lamellar grating i.e. in the geometry to be considered the refractive index is a step function. This refractive index has to be expanded into a sum of weighted basis functions in order to solve the Maxwel equations using a method of moments. One usually uses a discrete set of Fourier functions as basis and test functions (Moharam and al. 1986 ), (Lalanne 1995), (Granet 1996). However, it is well known that the expansion of sharp functions into Fourier series needs a large number of terms to provide a good description of the refractive index function and of sharp variation of the electromagnetic field.

It is known in the signal or image processing applications that multiresolution analysis is a way to overcome this issue. The principle is to analysis a local discontinuity of the field or the refractive index function using different scales. Applying a multiresolution expansion to the field and the refractive index function consist in an expansion on a scaling function at a lowest resolution, plus wavelets at a lowest resolution subsequent higher resolution. This multiresolution analysis is equivalent to an expansion that were using only the highest resolution basis. However in this paper, we describe only this latter expansion and view it as a preliminary step towards multiresolution. Namely the refractive index and the electromagnetic field are expanded here on one scale of basis function.

\section{Formulation}

In this section, we derive an operator that takes into account the inhomogeneity of the medium. For sake of simplicity, we assume 1D features and TE polarization (the problem is invariant according to the z-direction). Of course, the method holds for TM polarization, which will not be described in this paper. We consider Maxwel's curl equations for 1D homogeneous or inhomogeneous isotropic media:

$$
\begin{aligned}
& Z_{0} H_{x}=\frac{i}{k} \frac{\partial E_{z}}{\partial y} \\
& Z_{0} H_{y}=\frac{i}{k} \frac{\partial E_{z}}{\partial x} \\
& v^{2}(x) H_{x}=\frac{i}{k}\left[\frac{\partial Z_{0} H_{x}}{\partial y}-\frac{\partial Z_{0} H_{y}}{\partial x}\right]
\end{aligned}
$$

where $E_{z}, H_{x}, H_{y}$ are the non-zero Cartesian components of the electric and magnetic field, respectively. $Z_{0}$ the vacuum impedance and $v(x)$ the refractive index. From these equations, it can be deduced that the electromagnetic field $E_{z}$ satisfies the Helmholtz equation:

$$
\left[\frac{\partial^{2}}{\partial x^{2}}+\frac{\partial^{2}}{\partial y^{2}}+k_{0}^{2} \varepsilon(x)\right] E_{z}(x, y)=0
$$

where $\varepsilon(x)=v^{2}(x)$ is the dielectric function. This function can be expanded as $\varepsilon(x)=\varepsilon+\Delta \varepsilon(x), \varepsilon$ being a constant.

Eq.(2) can be rewritten in the following form:

$$
\left[H_{0}+k_{0}^{2} \Delta \varepsilon(x)\right] E_{z}(x, y)=-\frac{\partial^{2} E_{z}(x, y)}{\partial y^{2}}
$$

where: 


$$
H_{0}=\left[\frac{\partial^{2}}{\partial x^{2}}+k_{0}^{2} \varepsilon\right]
$$

We seek solutions of the form $E_{z}(x, y)=\chi(x) \kappa(y)$, and we assume a complex exponential dependance of the field in the $y$-direction $\kappa(y)=\exp \left(-i k_{0} r y\right)$. Thus we get an eigenvalue problem:

$$
\left[H_{0}+k_{0}{ }^{2} \Delta \varepsilon(x)\right] \chi(x)=k_{0}^{2} r^{2} \chi(x)
$$

In the following, we will adopt the "braket" notation $(\langle\bullet \mid \cdot\rangle)$.

A moment method using the Galerkin scheme is used to solve numerically the above eigenvalue equation (6). In our investigation, the basis and test functions $\left|\Phi_{k}^{N}(x)\right\rangle$ are spline functions. They show the advantage of having a compact support. $N$ denotes the scale factor, it controls the dilation of the scaling function $\left|\Phi_{k}^{N}(x)\right\rangle$ and $k$ relates to the position of the test function. For a given value of $N, \Phi_{k}^{N}(x)=\Phi^{N}(x-k)$.

$\left|\chi^{N}\right\rangle$ and $\left|\Delta \varepsilon^{N}(x)\right\rangle$ are expressed as follows:

$$
\left|\chi^{N}(x)\right\rangle=\sum_{k} \varphi_{k}^{N}\left|\Phi_{k}^{N}(x)\right\rangle, \quad \text { and }\left|\Delta \varepsilon^{N}(x)\right\rangle=\sum_{k} \varepsilon_{k}^{N}\left|\Phi_{k}^{N}(x)\right\rangle
$$

By substituting Eq.(7) in Eq.(6), and projecting on $\left\langle\Phi_{n}^{j}(x)\right|$, the eigenvalue problem becomes:

$\frac{1}{k_{0}^{2}} \sum_{m}\left\langle\Phi_{n}^{N} \mid \Phi_{m}^{{ }^{N}}\right\rangle \varphi_{m}^{N}+\sum_{m} \varepsilon\left\langle\Phi_{n}^{N} \mid \Phi_{m}^{N}\right\rangle \varphi_{m}^{N}+\sum_{p, m}\left\langle\Phi_{n}^{N} \mid \Phi_{p}^{N} \Phi_{m}^{N}\right\rangle \varepsilon_{p}^{N} \varphi_{m}^{N}=r^{2} \sum_{m} \varphi_{m}^{N}\left\langle\Phi_{n}^{N} \mid \Phi_{m}^{N}\right\rangle$

$\Phi_{n}{ }_{n}^{N}$ denotes the second derivative of $\Phi_{n}^{N}$ with respect to $x$.

Thus we get the following matrix relation:

$$
\left[\frac{1}{k_{0}^{2}} D+G+M\right]\left[\varphi_{q}^{N}\right]=r_{q}^{N 2} G\left[\varphi_{q}^{N}\right]
$$

with:

$$
D=\left(\begin{array}{ccc}
\left\langle\Phi_{0}^{N} \mid \Phi_{0}^{\prime N}\right\rangle & \ldots & \left\langle\Phi_{0}^{N} \mid \Phi_{N}^{\prime N}\right\rangle \\
\vdots & \ddots & \vdots \\
\left\langle\Phi_{N}^{N} \mid \Phi_{0}^{\prime N}\right\rangle & \cdots & \left\langle\Phi_{N}^{N} \mid \Phi_{N}^{\prime N}\right\rangle
\end{array}\right), \quad G=\left(\begin{array}{ccc}
\left\langle\Phi_{0}^{N} \mid \Phi_{0}^{N}\right\rangle & \ldots & \left\langle\Phi_{0}^{N} \mid \Phi_{N}^{N}\right\rangle \\
\vdots & \ddots & \vdots \\
\left\langle\Phi_{N}^{N} \mid \Phi_{0}^{N}\right\rangle & \cdots & \left\langle\Phi_{N}^{N} \mid \Phi_{N}^{N}\right\rangle
\end{array}\right)
$$

and

$$
M=\left(\begin{array}{c}
\cdots \\
\vdots \quad \int \varepsilon(x) \Phi_{n}^{N}(x) \Phi_{p}^{N}(x) d x \quad \\
\cdots
\end{array}\right)
$$

$\left[\varphi_{q}^{N}\right]$ is a column vector. We can associate to any eigenvalue $r_{q}$ an eigenmode:

$$
F_{q}^{N}(x, y)=\exp \left(-i k_{0} r_{q}^{N} y\right) \sum_{n} \varphi_{n q}^{N} \chi_{n}^{N}(x)
$$

Thus the general solution at the $N^{\text {th }}$ scale level is written as a linear combination of the eigenfunctions:

$$
E_{z}^{N}(x, y)=\sum_{q} R_{q}^{N} F_{q}^{N}(x, y)
$$

Two sets of modes are observed:

1. those propagating or decaying in the positive $y$ direction: 


$$
\left\{r_{q}^{N+}\right\}=\left\{r_{q}^{N} /\left(r_{q}^{N} \in \mathbb{R}^{+}\right) \quad \text { or } \quad\left(r_{q}^{N} \in \mathbb{C} \& \operatorname{Im}\left(r_{q}^{N}\right)<0\right)\right\}
$$

2. and those traveling in the opposite direction:

$$
\left\{r_{q}^{N-}\right\}=\left\{r_{q}^{N} /\left(r_{q}^{N} \in \mathbb{R}^{-}\right) \quad \text { or } \quad\left(r_{q}^{N} \in \mathbb{C} \& \operatorname{Im}\left(r_{q}^{N}\right)>0\right)\right\}
$$

The electromagnetic field is then expressed under the following form:

$$
E_{z}^{N}(x, y)=\sum_{n} \chi_{n}^{N}(x) \sum_{q}\left[R_{q}^{N+} \varphi_{n q}^{N+} \exp \left(-i k_{0} r_{q}^{N+} y\right)+R_{q}^{N-} \varphi_{n q}^{N-} \exp \left(-i k_{0} r_{q}^{N-} y\right)\right]
$$

The field coefficients $R_{q}^{N-}$ and $R_{q}^{N+}$ are determinated by the boundary conditions of continuity of the tangential component of the fields at the interfaces. For that purpose, the tangential component of the magnetic field $H_{x}(x, y)$, which is needed for these boundaries conditions is drawn from Maxwell equation as follows:

$$
H_{x}^{N}(x, y)=\sum_{n} \chi_{n}^{N}(x) \sum_{q}\left[R_{q}^{N+} \varsigma_{n q}^{N+} \exp \left(-i k_{0} r_{q}^{N+} y\right)+R_{q}^{N-} \varsigma_{n q}^{N-} \exp \left(-i k_{0} r_{q}^{N-} y\right)\right](12-\mathrm{a})
$$

where

$$
\left[\varsigma_{n q}^{N}\right]=\left[\varphi_{n q}^{N}\right]\left[r_{n q}^{N}\right], \text { with }\left[r_{n q}^{N}\right]=\operatorname{diag}\left(r_{n q}^{N}\right)
$$

\section{Nodal B-Spline Expansion}

We have chosen to use B-spline as basis functions. The first order B-spline function is defined as following:

$$
\Phi_{k}^{N}(x)=\frac{1}{\left\langle\Phi_{k}^{N}(x) \mid \Phi_{k}^{N}(x)\right\rangle} \begin{cases}x-\frac{k d}{N} & x \in[k, k+1] \frac{d}{N} \\ -x+\frac{(k+2) d}{N} & x \in[k+1, k+2] \frac{d}{N}\end{cases}
$$

where $d$ denotes the size of the analysis domain.

The number of the basis $\left|\Phi_{k}(x)\right\rangle_{k}$ elements that we need to describe the whole space from 0 to $d$ is $N$ for a perfectly conducting boundary condition in the $\mathrm{x}$-direction $(k \in[0, N-1])$. For non-zero boundary conditions, it takes $N+2$ elements to describe properly the field at the boundaries $(k \in[-1, N])$. An example of first order spline basis is shown in figure 2 . For this kind of polynomial type basis functions, all the scalar product can be computed analytically. We get:

$$
\begin{aligned}
& \left\langle\Phi_{k}(x) \mid \Phi_{k}(x)\right\rangle=1 \\
& \left\langle\Phi_{k}(x) \mid \Phi_{k+1}(x)\right\rangle=\left\langle\Phi_{k-1}(x) \mid \Phi_{k}(x)\right\rangle=\frac{1}{4} \\
& \left\langle\Phi_{k}(x) \mid \Phi_{k^{\prime}}(x)\right\rangle=0 \quad \text { otherwise }
\end{aligned}
$$

In the case of periodic boundary conditions, additional contraints are put on the side elements:

$$
\left\langle\Phi_{k}(x) \mid \Phi_{k^{\prime}}(x)\right\rangle=\left\langle\Phi_{k}^{\prime}(x) \mid \Phi_{k^{\prime}}^{\prime}(x)\right\rangle \text {, with } \Phi_{k}^{\prime}(x)=\frac{d \Phi_{k}(x)}{d x} \text { and } \Phi^{\prime \prime}{ }_{k}(x)=\frac{d^{2} \Phi_{k}(x)}{d x^{2}}
$$

The periodic condition at $x=d$ and $x=0$ may be numerically translated onto the following form:

$$
\begin{aligned}
& \left\langle\Phi_{N-1}(x) \mid \Phi_{-1}(x)\right\rangle=\left\langle\Phi_{-1}(x) \mid \Phi_{N-1}(x)\right\rangle=\left\langle\Phi_{-1}(x) \mid \Phi_{-1}(x)\right\rangle, \\
& \left\langle\Phi_{N-2}(x) \mid \Phi_{-1}(x)\right\rangle=\left\langle\Phi_{-1}(x) \mid \Phi_{N-2}(x)\right\rangle=\left\langle\Phi_{-1}(x) \mid \Phi_{0}(x)\right\rangle
\end{aligned}
$$

These relations are also valid for $\left|\Phi^{\prime}{ }_{k}(x)\right\rangle$ since the derivative have also to be periodic.

In order to improve the accuracy of the expansion. We also used second order B-spline nodal basis function which are defined as follows: 


$$
\Phi_{k}(x)=\frac{3}{N} \begin{cases}\frac{1}{2} x^{2}-2 \frac{k d}{N} x+\left(\frac{k d}{N}\right)^{2} & x \in \frac{d}{N}\left[\begin{array}{ll}
k & k+1
\end{array}\right] \\
-x^{2}+2 \frac{d}{N}\left(k+\frac{3}{2}\right) x-\left(\frac{d}{N}\right)^{2}\left(\left(k+\frac{3}{2}\right)^{2}-\frac{3}{4}\right) & x \in \frac{d}{N}\left[\begin{array}{ll}
k+1 & k+2
\end{array}\right] \\
\frac{1}{2} x^{2}-2 \frac{d}{N}(k+3) x+\left(\frac{d}{N}(k+3)\right)^{2} & x \in \frac{d}{N}\left[\begin{array}{ll}
k+2 & k+3
\end{array}\right]\end{cases}
$$

In this case, the previously described periodic conditions must include in addition the kets $\left|\Phi_{N \max }(x)\right\rangle,\left|\Phi_{2}(x)\right\rangle,\left|\Phi_{N \max -2}(x)\right\rangle$ and $\left|\Phi_{-2}(x)\right\rangle$. The number of the basis $\left|\Phi_{k}(x)\right\rangle_{k}$ elements that we need to describe the whole space from 0 to $d$ is here $\mathrm{N}+4$ for non-zero boundary condition $(k \in[-2, N+1])$. as illustrated in figure 3 .

\section{Application to EUV lithography mask}

The above formalism is applied to an extreme UV lithography mask. We consider in the present paper a gaussian defect perfectly replicated along the multilayer as illustratred in figure 1 . The defect height is $7 \mathrm{~nm}$ and its width is $60 \mathrm{~nm}$. For that purpose, the "blank" is divided in 600 sublayers which refractive index is a succession of step functions. The handling of the boundary conditions is done using a S-matrix scheme (G. Granet 2001). The reflected intensity (P. Schiavone and al. 2001), is computed at the top of the blank from the expression of Eq (11-b).

The figure 4 presents the convergence results obtained from a first, second and third order B-spline nodal basis, for differents values of the truncation order $\mathrm{N}$. The computed diffracted efficiencies are in a good agreement with those obtained from the MMFE (Modal Method by Fourier Expansion) and FDTD (Finite Difference method in Time Domain), (M. Besacier and al. 2003). Nevertheless figure 5 shows that the RCWA and the quadratic B-spline nodal basis are performing the best. On this figure, we represent the convergence of the total normalised reflective power, versus to the truncation order. The MMSNE offers certain advantages such as the implementation of a non-periodic boundary conditions in the x-direction, this is not possible with the MMFE because of the natural periodicity of the basis function. Other advantage of the MMSNE is due to the compact support of the basis elements. This property allows the implementation of a local multi-level analysis that will be presented in a future paper.

\section{Conclusion}

In this paper, we have adapted a B-spline expansion to a modal method to simulate a defect in EUV mask. The global conception of the MMSNE is the same as the other modal methods such as Cmethod and MMFE: we solve in an eigenvalue problem and the solutions are matched using a S-matrix formalism. The fundamental difference between these three methods is the resolution of the eigenvalue equation. Both C-method and MMFE are implemented with a Fourier based expansions although their eigenvalue equation are differents. The MMSNE and the MMFE have the same eigenvalue equation whereas they are implemented with different basis functions. However the Fourier expansion in not adapted to handle abrupt edges, for example the absorber pattern on the EUV lithography mask and it is well kwnon that a wavelet analysis is more efficient to described these kind of discontinuty.

Although the structure is treated here without using the multiresolution analysis, the MMSNE is already competitive compared to the MMFE. Nevertheless, the addition of a hierarchical basis will allow to implement a multi-level analysis, preferably located around the fast variation of the field. Thus the MMSNE will certainly be performing better than the MMFE. This next step of our work will be presented in a subsequent paper. 


\section{References}

M. G. Moharam and T. K. Gaylord. J. Opt. Soc. Am. A 3 (11) 780, 1986.

P. Lalanne, G. Morris, J. Opt. Soc. Am. A 3 (4) 1780, 1996.

G. Granet, B Guizal, J. Opt. Soc. Am. A 13 (9) 779, 1997.

G. Granet, Optical and Quantum Electronics 33 471, 2001.

P. Schiavone, G. Granet, J. Y. Robic, Microelectronic Engineering 57-58, 497, 2001.

M. Besacier, P. Schiavone, G. Granet, V. Farys, Proc SPIE conf. Energing Lithographic technologies VII, Vol 5037, P 822, 2003. 


\section{Figures caption:}

Figure 1: Shematic of a multilayer mirror with a gaussian defect.

Figure 2: Set of a first order B spline used for expansion of field with non-zero periodic boundary condition.

Figure 3: Set of a second order B spline used for expansion of field with non-zero periodic boundary condition.

Figure 4: Intensity reflected from a defective EUV mask blank at $315 \mathrm{~nm}$ from the mirror surface: solid line: MMSNE with third order B-spline, dashed line: MMSNE with second order B-spline, dotted line: MMSNE with first order B-spline,

Figure 5: Total reflected power vs troncation order: solid line: MMSNE with third order B-spline, dashed line: MMSNE with second order B-spline, dash-dotted line: MMSNE with first order B-spline, dotted line: MMFE 


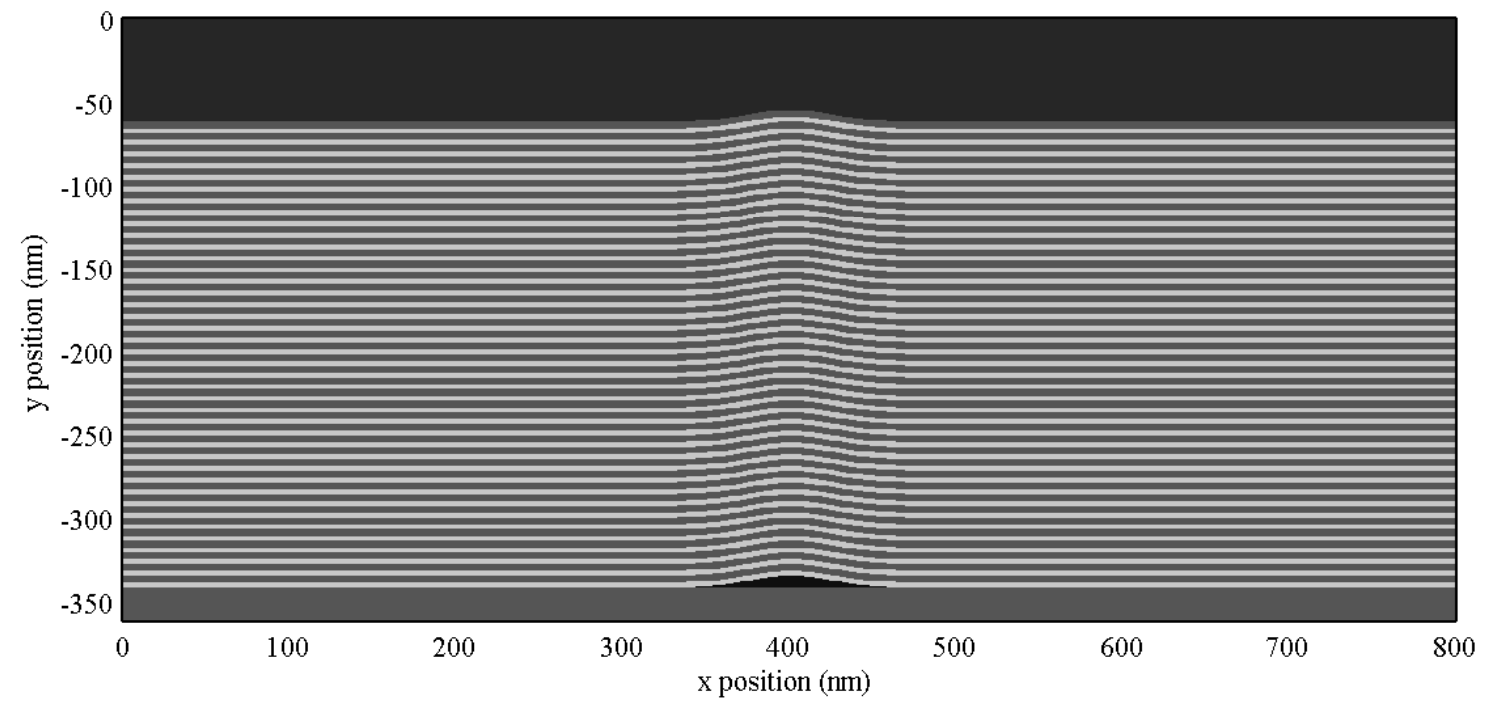

Figure 1 


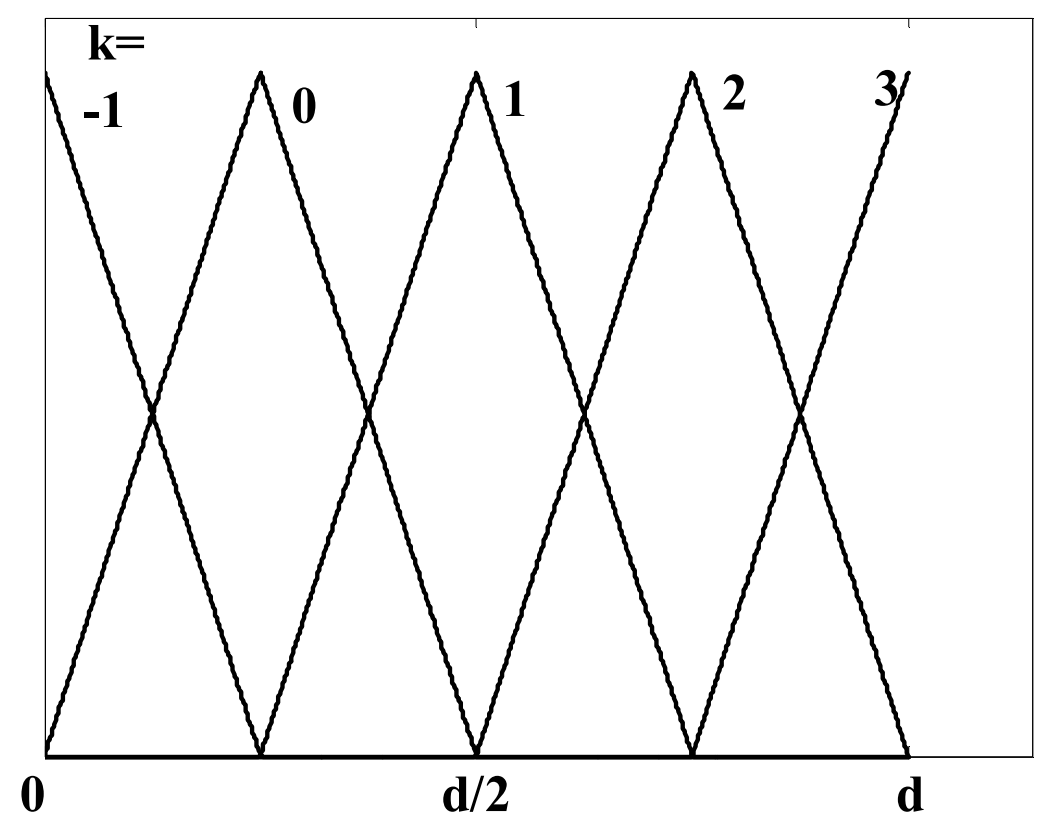

Figure 2

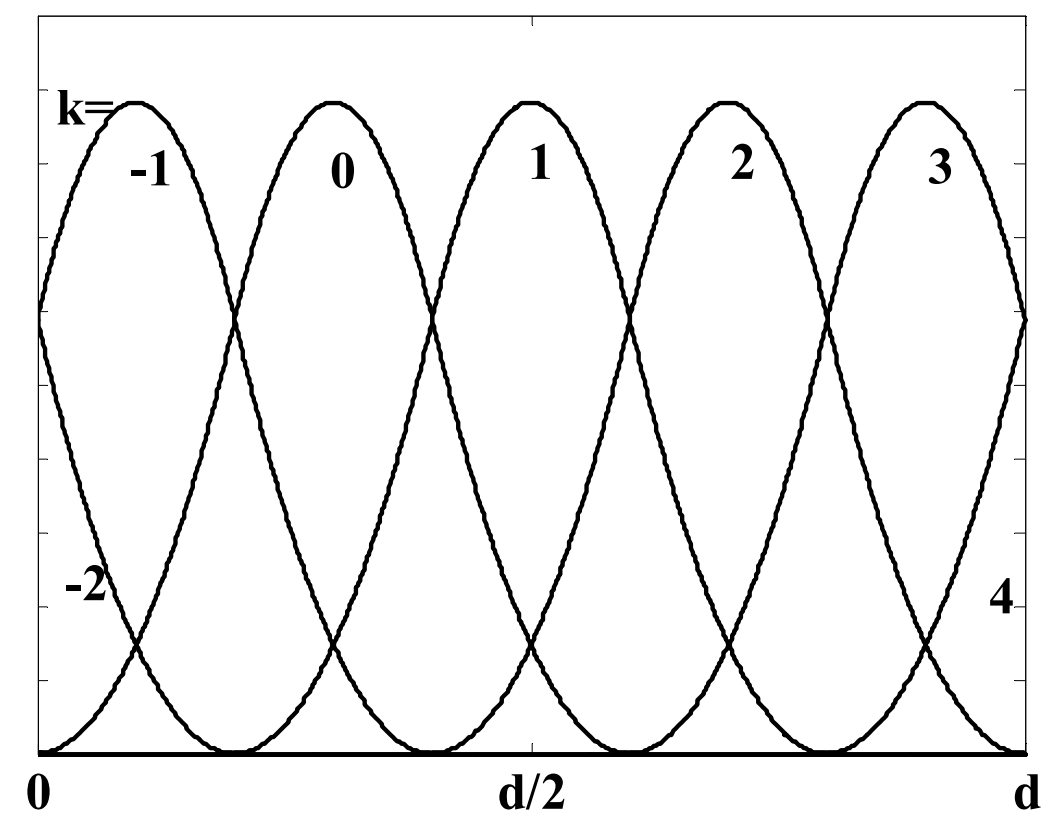

Figure 3 


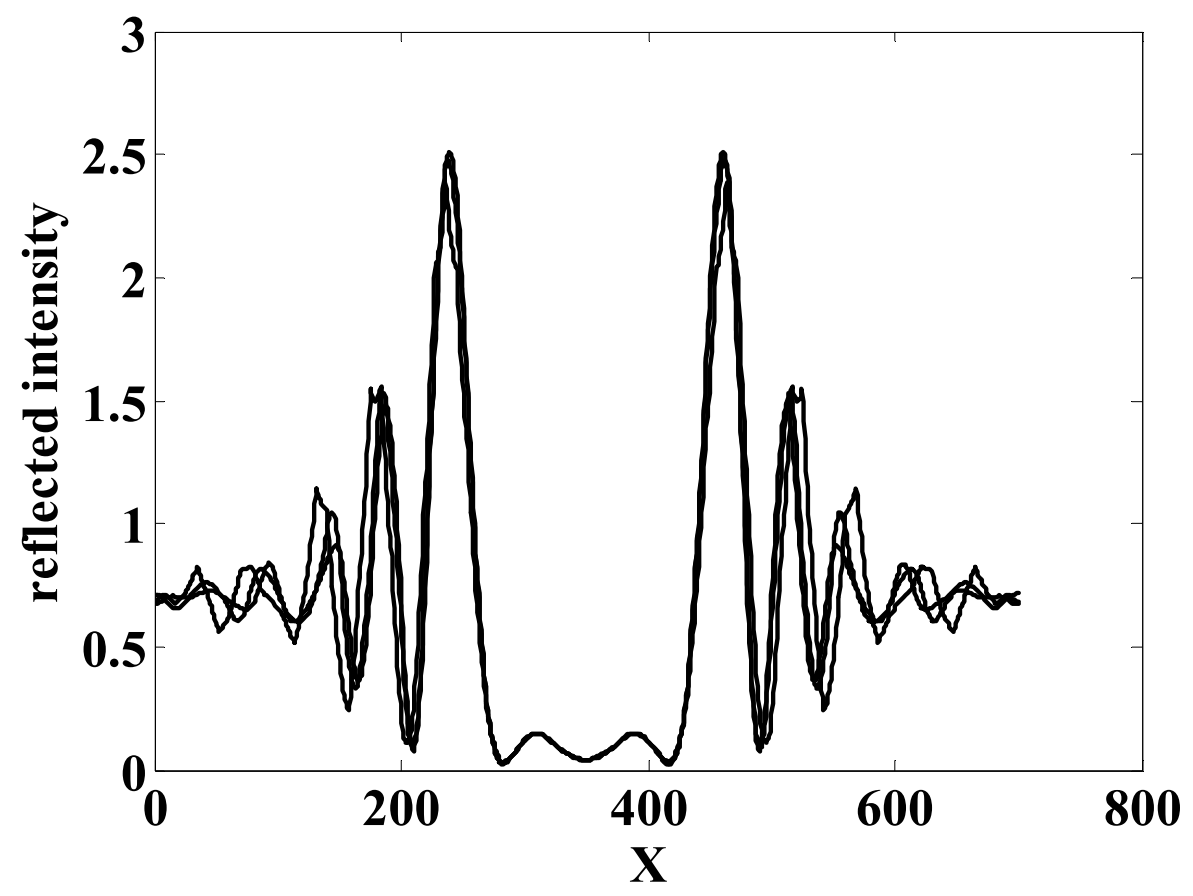

Figure 4

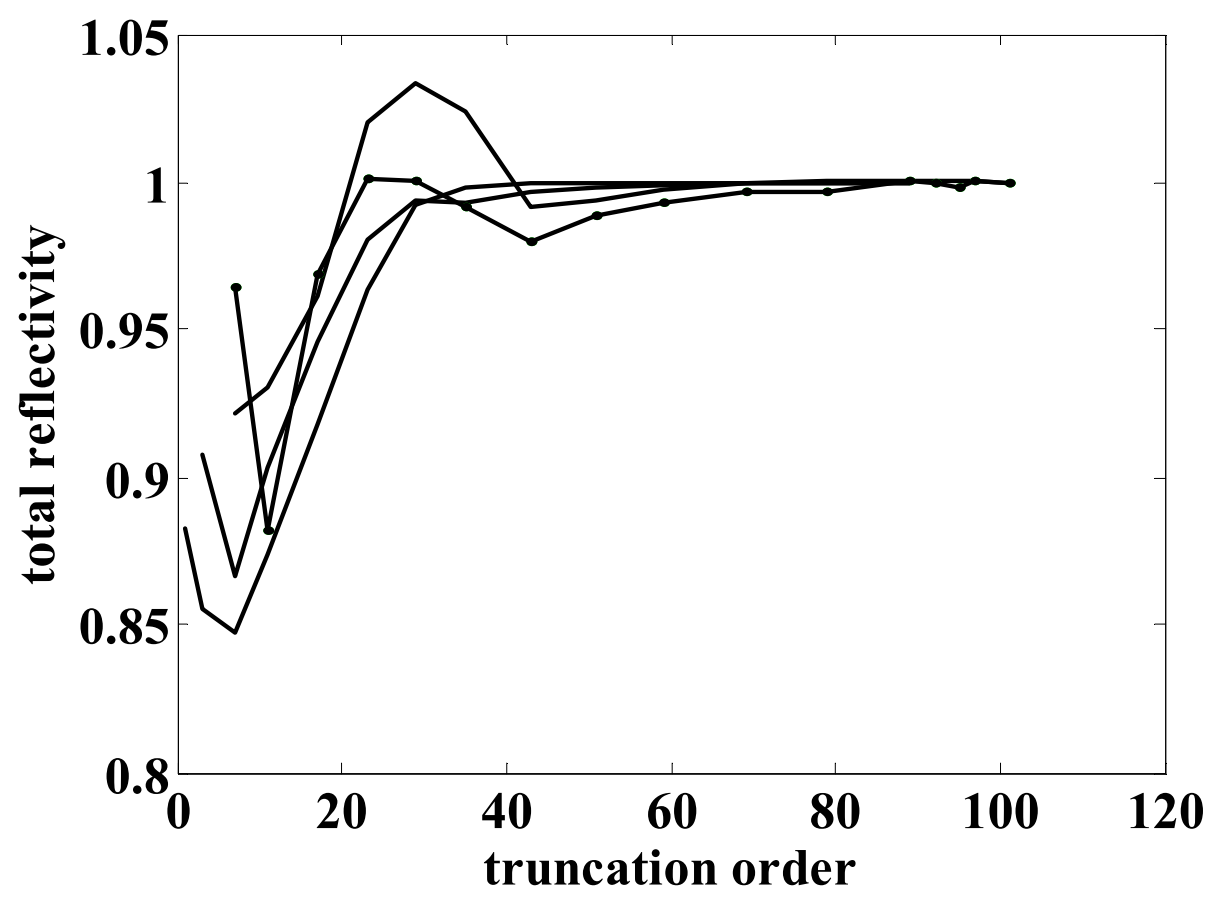

Figure 5 